\title{
van der Waals epitaxy of soft twisted bilayers: Lattice relaxation and static distortion waves
}

Cong Jin, ${ }^{\dagger, \star}$ Brian C. Olsen, ${ }^{\dagger, \downarrow}$ Erik J. Luber, ${ }^{*},+\neq$ Jillian M. Buriak $*,+, \neq$

'Department of Chemistry, University of Alberta, 11227 Saskatchewan Drive, Edmonton, AB T6G 2G2, Canada.

National Research Council Canada, Nanotechnology Research Centre, Edmonton, AB T6G 2M9, Canada.

*E-mail: eluber@ualberta.ca

*E-mail: jburiak@ualberta.ca 


\begin{abstract}
Interfaces comprising incommensurate or twisted hexagonal lattices are ubiquitous and of great interest. From adsorbed organic/inorganic interfaces in electronic devices, to superlubricants, and more recently to van der Waals bilayer heterostructures (vdWHs) of graphene and other 2D materials that demonstrate a range of remarkable properties such as superconductivity and ferromagnetism. Here we show how growth of 2D crystalline domains of soft block copolymers (BCPs) on patterned hard hexagonal lattices provide fundamental insights into van der Waals heteroepitaxy. At moderate registration forces, it is experimentally found that these BCP-hard lattice vdWHs do not adopt a simple Moiré superstructure, but instead adopt local structural relaxations known as static distortion waves (SDW). Simulations reveal that SDWs are a primary mechanism of energy minimization, and are the origin of the observed preferential twist angle between the lattices.
\end{abstract}
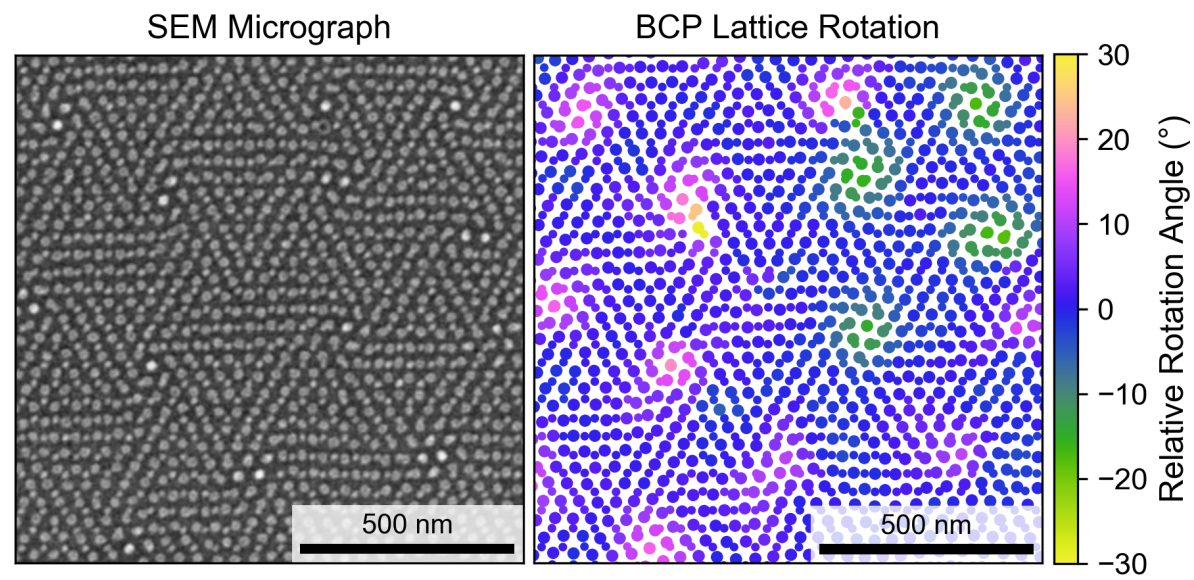

\title{
Graphical Abstract
}




\section{Main}

Epitaxial engineering of thin film devices has been a critically important feature of many modern optoelectronic devices, where the interfacial registration forces between two different materials can be used to modify structural, electrical and optical properties. ${ }^{1-3}$ Rational tuning of material properties via epitaxy is being increasingly applied to socalled 2-dimensional van der Waals or soft materials for applications such as switchable ferromagnets, ${ }^{4-7}$ tunable field effect and logic devices, ${ }^{2,8-13}$ volatile organics sensors, ${ }^{14}$ high-efficiency photodetectors, ${ }^{15,16}$ quantum communication devices ${ }^{17}$ and superlubricity. ${ }^{18}$ Since the discovery of superconductivity in twisted magic-angle graphene bilayers by Jarillo-Herrero and co-workers in $2018,{ }^{19}$ there has been an explosion of research into understanding the properties of twisted bilayer van der Waals heterostructures . ${ }^{1,20-26}$ Critical to the functional properties of these 2-dimensional van der Waals bilayer heterostructures is the local atomic structure and orientation that they adopt upon epitaxial registration, which can significantly influence electronic and magnetic properties..$^{20,27-29}$ The epitaxial structure and orientation of 2-dimensional van der Waals bilayer heterostructures, including twisted magic-angle graphene bilayers, has been explained by the conceptually and mathematically simple 20 -year-old model of superposition of plane waves, resulting in well-defined superstructure patterns. ${ }^{30}$ However, in 2016 it was experimentally shown through ultra-precise scanning tunneling microscopy (STM) by Fritz and co-workers, ${ }^{31}$ that this model is nuanced. These experiments confirmed the existence of periodic structural relaxations called static distortion waves (SDW), which were predicted by McTague and Novaco in 1977. ${ }^{32} \mathrm{An}$ experimental understanding of structural relaxation in twisted van der Waals heterointerfaces is only in its infancy, as the preparation and subsequent measurement of local atomic/molecular displacements is challenging, ${ }^{33}$ where it has been shown that there can be significant atomic relaxations that alter the electronic properties beyond a simple Moiré band description. ${ }^{20}$ Moreover, understanding the effects of the inter-to-intralayer forces and relative pitch ratio between layers is difficult to probe experimentally, as these parameters are generally highly constrained (as is the case for carbon-carbon bonds, for instance). In this work we combine electron beam lithography (EBL) with block 
copolymer (BCP) self-assembly, which allows for facile and precise tuning of the interto-intralayer forces and relative pitch ratio of a van der Waals bilayer heterostructure. Moreover, the characterization of these heterostructures can be performed using scanning electron microscopy (SEM). The pitch ratio between the BCP lattice and e-beam-derived post array was systematically varied from 0.68 to 2.05 , resulting in 20 unique pitch ratios. In this work it is found that SDWs are ubiquitously and easily observed at most incommensurate pitch ratios. Simulations are used to show that the observed preferential angles and superstructures are a result of SDWs dominating the energy minimization of the van der Waals bilayer configuration. 


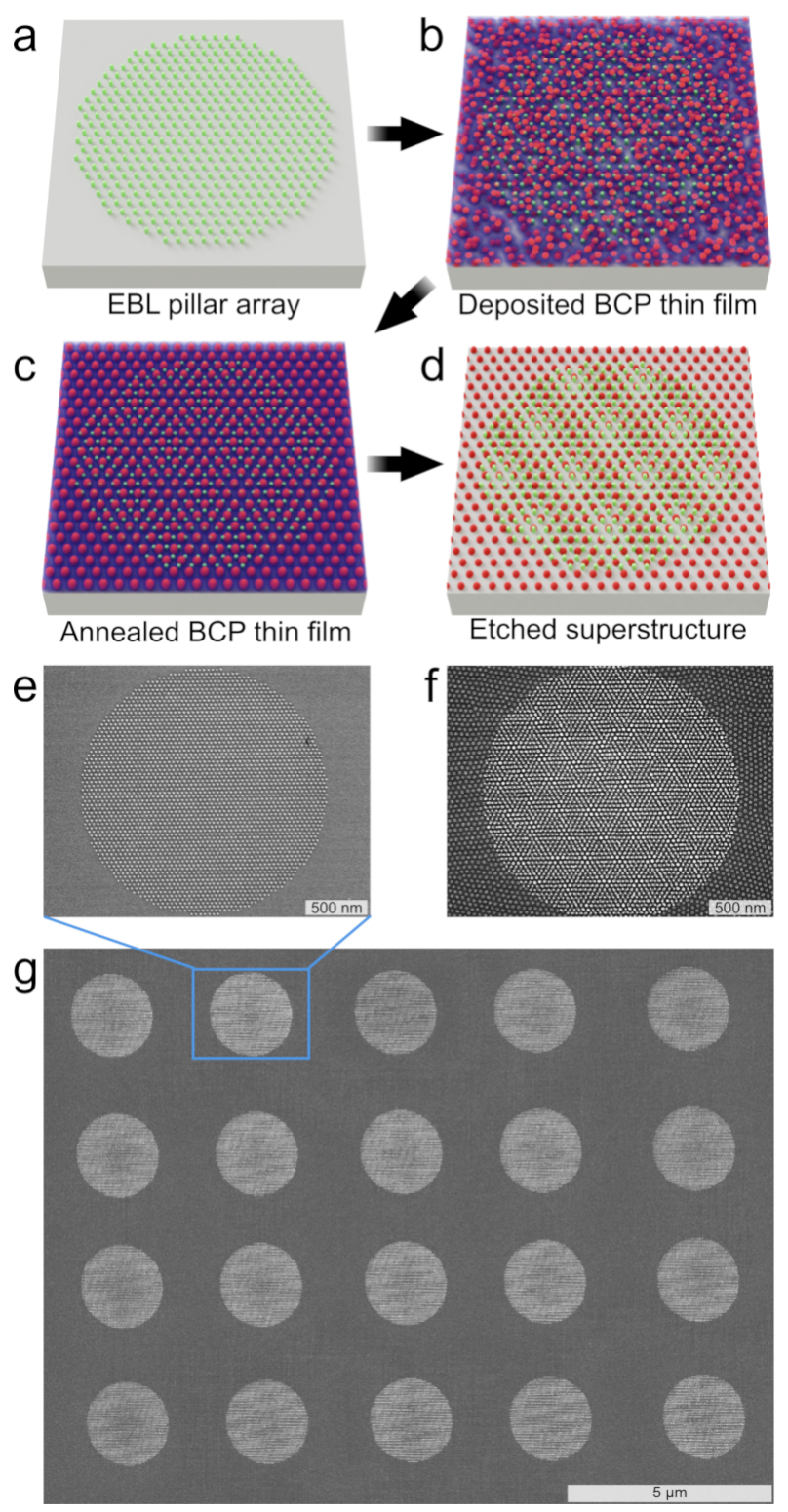

Figure 1. Schematic illustration for the fabrication of tunable van der Waals heterostructures using EBL and BCP self-assembly. a, Circular array of hexagonally ordered silica posts fabricated by EBL. b, Deposition of a thin film of a PS- $b$-PDMS BCP on the EBL patterned post array. c, Annealing of the thin film of PS- $b$-PDMS, resulting in a hexagonally packed dot array, overlayed on the silica posts. d, Reactive ion etching (RIE) of the annealed BCP, producing an all-silica superstructure. e, SEM micrographs of a circular EBL post array and a $\mathbf{g} 4 \times 5$ grid of identical EBL post arrays. f, SEM micrograph of the resultant superstructure comprising the incommensurate $\mathrm{BCP}$ - and EBL-derived post arrays.

Figure 1 illustrates how the post array templates prepared using EBL were used to systematically interrogate the registration and epitaxy of a dot-forming BCPs on a nonplanar surface with periodic topography. In this manner, the effects of the relative pitch 
ratio (post pitch/BCP pitch) could be easily adjusted over a wide range. Circular arrays of hexagonally ordered posts were first fabricated via EBL (Figure 1a and Figure 1e), HSQ resist to produce silica posts on a flat silicon surface. Thin films of dot-forming BCPs, PS- $b$-PDMS(31K-14.5K)/30 wt $\%$ PS, with a native pitch of $\sim 44 \mathrm{~nm},{ }^{34}$ were spin-cast on these patterned silicon surfaces (Figure 1b) and solvent annealed in THF vapor, ${ }^{34}$ forming hexagonally packed dot arrays (Figure 1c). The annealed BCP thin films were then etched in an $\mathrm{O}_{2} / \mathrm{CF}_{4}$ plasma, which transformed the PDMS domains into silica features, resulting in the emergence of an all-silica superstructure comprising the EBL post and BCP dot arrays (Figure 1d and f). 15 to 20 identical circular post arrays were produced for each of the 20 different dot arrays, as shown in Figure 1e and g, to enable statistically meaningful quantification of these superstructure patterns. The pitch of the hexagonally ordered EBL post arrays ranged from 30 to $90 \mathrm{~nm}$ (nominal pitch ratios from 0.68 to 2.05 ), in increments ranging from 2 to $6 \mathrm{~nm}$. Of the 20 pitch ratios explored in this work, 17 of them are incommensurate, and 3 are commensurate (pitch ratios of $1, \sqrt{3}$ and 2), ${ }^{35}$ corresponding to the 44,76 and $88 \mathrm{~nm}$ pitch EBL templates. EBL post arrays were fabricated with a nominal post height of $5.0 \mathrm{~nm}$ to match the height of silica dots used in our previous work. ${ }^{36}$ The post height was set by adjusting HSQ film thickness before electron beam exposure, as well as additional etching after electron beam exposure (see Methods for more detail). EBL post heights were confirmed and measured by AFM (Figure S1).

From our previous work, ${ }^{36}$ it was expected that the epilayer BCP lattice would adopt a preferential twist angle to the e-beam post lattice. In order to measure the twist angle for a given pitch ratio, first the local relative rotation of each BCP dot is measured, which is simply the average angular rotation of its six nearest neighbors (relative to the [10] direction of the bottom layer lattice). Given that a single post array typically interacts with 1-3 BCP grains, it was necessary to acquire relative rotation data from a large number of post arrays to statistically verify the existence of preferential orientations. As such, large-area high-magnification scanning helium ion microscopy (HiM) images were acquired over an entire array of 20 EBL patterned regions. Large-area mappings of the absolute relative rotation (and corresponding histograms) for pitch ratios of 0.82 and 1.13 are shown in Figure S2, which clearly shows that the patterned regions adopt a 
preferential twist orientation relative to the unpatterned planar regions. It is noted that for pitch ratios close to 1.0 the twist angle is near $0^{\circ}$, while at pitch ratios near $\sqrt{3} \approx 1.73$ the twist angle is near $30^{\circ}$, and then transitions back to $0^{\circ}$ at pitch ratios near 2.0 (see Figure S3).

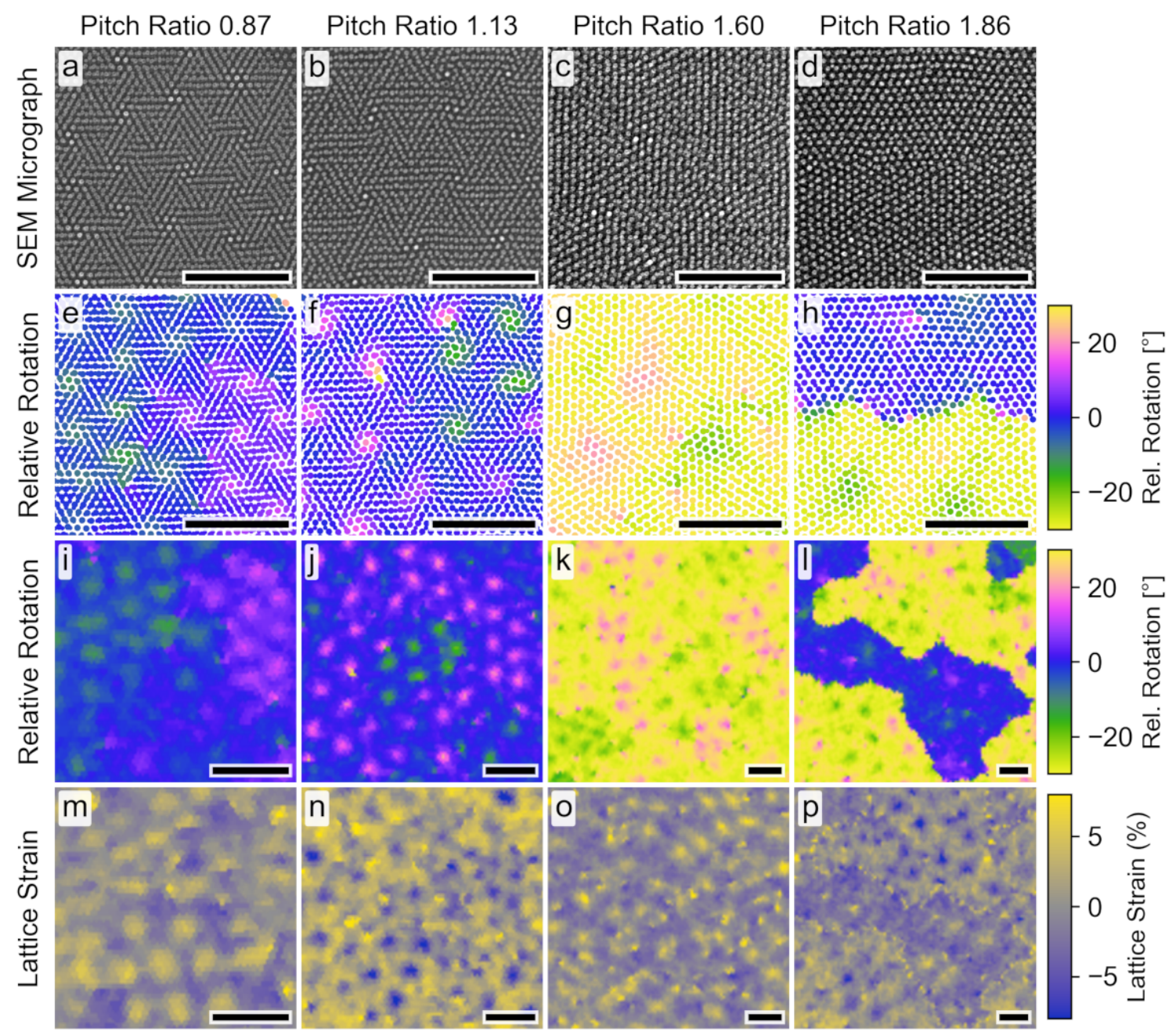

Figure 2. Epitaxial configurations of BCP lattices registered to EBL post arrays at different pitch ratios. a-d, SEM micrographs of samples at various pitch ratios as indicated. $\mathbf{e}-\mathbf{h}, \mathrm{BCP}$ dot maps showing the local rotation angle of the BCP lattice and the positions of the dots and e-beam posts at the same locations as the SEM micrographs above. $\mathbf{i}-\mathbf{l}$, Larger area dot maps showing local lattice rotation angle. $\mathbf{m}-\mathbf{p}$, Dot maps showing local lattice strain at the same location as the large scale angle maps above. All scale bars are $500 \mathrm{~nm}$.

Shown in Figure 2 are representative SEM micrographs of the resultant epitaxial configurations at different pitch ratios (see Figures S4, S5 for all pitch ratios). Inspection of these images reveals a large diversity of structures, ranging from superstructures 
consisting of large triangular-like domains (Figure 2a,b), to flower-like domains (Figure $2 \mathrm{c}, \mathrm{d}$ ). To better understand the complex registration behavior of the BCP dot lattice as a function of pitch ratio, these configurations were quantified using the metrics of relative rotation angle and lattice strain. The lattice strain is defined as the percent change of the local BCP pitch, relative to the unstrained BCP pitch in the unpatterned regions directly proximal to the EBL patterned region (see Supplementary Information for more details). Inspection of the relative rotation maps (Figure 2e-h) reveals an unexpected and interesting phenomenon, in addition to the observed global preferential orientation of the BCP lattices. At pitch ratios of 0.87 and 1.13, there are very obvious pink and green nodes in Figure 2e-f that correspond to localized lattice rotations of $\pm 10^{\circ}$ $15^{\circ}$. A similar observation is also found near the commensurate pitch ratio of $\sqrt{3} \simeq 1.73$. Despite a significant change in the preferential angle of the BCP lattice to $\sim 30^{\circ}$, there still appears to be localized rotational nodes, which can be seen in Figure 2k,1. Viewing these rotational maps at larger length scales (where the individual dots are no longer visible, Figure $2 \mathrm{i}-1$ ) reveals that these rotational nodes are spatially ubiquitous over the entire patterned post array region and across different pitch ratios. Analysis of the lattice strain maps (Figure $2 \mathrm{~m}-\mathrm{p}$ ) reveals a similar pattern as the relative rotation maps. At a pitch ratio of 0.87 , there are nodes of localized extension and outside of these nodal regions the BCP lattice is strained compressively towards a commensurate pitch ratio of 1 . In accordance with this behavior, at a pitch ratio of 1.13 , there are compressional strain nodes which compensate for the BCP lattice straining in extension towards the commensurate pitch ratio of 1 outside of these nodes. Similar effects are also observed for pitch ratios of 1.60 and 1.86, which are above and below the commensurate pitch ratio of $\sqrt{3}$. Finally it is noted that this phenomenon of localized rotational and strain nodes in the BCP lattice is observed at almost all of the tested incommensurate pitch ratios (Figure S3 and Figure S6).

The existence of rotational nodes for incommensurate van der Waals monolayers was theoretically predicted in 1977 by Novaco and McTague. ${ }^{32}$ Specifically, it was predicted that extremely small but spatially coordinated and localized relaxation/perturbations from equilibrium lattice positions would minimize the free energy of incommensurate epitaxial configurations, which they termed static-distortion waves. Experimental evidence of 
SDWs has been inferred from low energy electron diffraction patterns of noble gas adlayers on graphite, ${ }^{37}$ and organic monolayers on metal surfaces. ${ }^{37-40}$ It was not until 2016, however, that Fritz and co-workers directly observed SDWs in real-space, using STM to detect sub-Angstrom displacements from equilibrium positions. ${ }^{31}$ These SDWs have also been predicted to occur in superconducting magic-angle twisted bilayer graphene and may play an important role in the functional properties of these topological materials..$^{20}$ Given the difficulties of directly characterizing these SDWs in real-space for atomic bilayer materials, $\mathrm{BCP}$ dot lattices on e-beam post arrays could be an ideal model system for studying the fundamental aspects of SDWs. Specifically, interlayer interactions can be systematically adjusted by varying the pitch and height of the e-beam posts.

To better understand the origins of SDW formation in soft-rigid incommensurate epitaxy, simulations were performed using LAMMPS molecular dynamics simulations. ${ }^{41}$ These simulations were performed using simple 2-dimensional pairwise potentials to approximate the relevant interactions that occur during incommensurate epitaxy of these van der Waals systems. Specifically, a modified Morse potential was used to model the top layer or epilayer intralayer interactions (e.g. the BCP layer), where the epilayer has a known equilibrium pitch with a hexagonal crystal structure (for a planar substrate). The intralayer interactions of the bottom layer are set such that it is perfectly rigid and does not move in response to the epilayer, which would be the case for an e-beam post array of silica features. Lastly, the interlayer potential is taken as a purely repulsive cosine potential, resulting in an energetic penalty for direct overlap between particles in the top and bottom layer, which decreases with separation distance. This form of the interlayer potential agrees with the model for BCP-BCP epitaxy proposed by Yager and coworkers, where the overlap between interblock regions and protrusions is maximized due to the relatively lower strain energy associated with stretching interblock regions compared to the intrablock regions. ${ }^{42}$ In general, these simplified potentials describe the most basic interactions for these epitaxial systems: the epilayer intralayer potential penalizes local structural relaxations/deviations within the layers, while the interlayer potential favors local structural relaxation in order to adopt commensurability between layers. ${ }^{43-47}$ In an epitaxially commensurate pair of lattices it is possible to achieve perfect 
closed-packing of the epilayer (minimizing the intralayer energy) while also minimizing the interlayer energy, where every particle in the top layer is residing in an interstitial position of the bottom layer. In an incommensurate system, it is not geometrically possible to independently minimize the inter- and intralayer energies, as such a compromise between close-packing and local relaxation/perturbations of the epilayer (assuming the bottom layer is perfectly rigid) must occur.
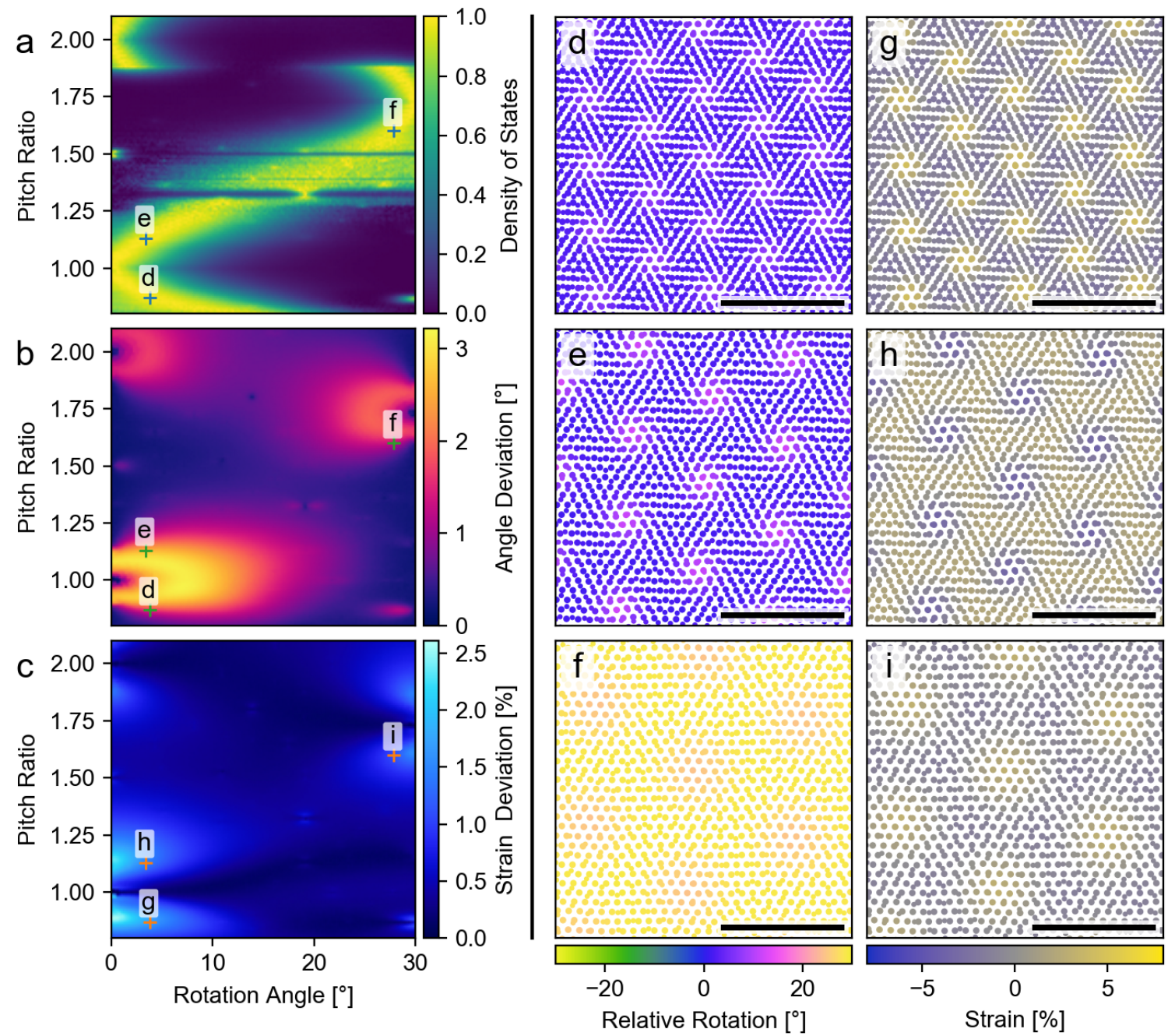

Figure 3. A summary of energy minimization calculations. Value maps of a, the rescaled density of states, encoded by global twist angle (for a fixed pitch ratio), b, the mean local angle deviation and $\mathbf{c}$, the mean strain deviation. $\mathbf{d}-\mathbf{f}$, Relaxed dot positions resulting from starting conditions as indicated in a. $\mathbf{d}, 3.8^{\circ}$ rotation and 0.87 pitch ratio. e, $3.4^{\circ}$ rotation and 1.13 pitch ratio. $\mathbf{f}, 27.9^{\circ}$ rotation and 1.60 pitch ratio. The color values represent the lattice angle at each dot with the color of the top lattice projected to the stationary bottom lattice for visualization. $\mathbf{g}-\mathbf{i}$, Dot map showing the same positions as df but color values indicate lattice strain at each dot. 
Using these simplified potentials the configurational density of states (DOS) is determined by allowing the system to relax for each pitch ratio as a function of initial twist angle, as shown in Figure 3a (see Simulation details section of the Methods section and Supplementary information for more details). For a given pitch ratio, the DOS is calculated by first determining the minimum configurational energy (defined as the total energy of the configuration divided by the maximum of the interlayer potential and the total number of posts) for all possible twist angles $\left(0^{\circ}-30^{\circ}\right)$, then converted to a probability distribution by assuming Boltzmann statistics. The DOS is also rescaled for each pitch ratio to increase contrast in the map (see Supplementary Information for details). The unscaled DOS and the configurational energy are shown in Figure S7a,b respectively. From this map of the configurational DOS, the experimental observation of a preferential twist angle can be reproduced. At pitch ratios near 1.0, configurations with a low-angle twist have the lowest energies and would be expected to occur at a higher frequency than high-angle twists. As the pitch ratio increases above 1.0, the twist angle of the lowest energy configuration increases, although there is also a significant increase in the breadth of the DOS. This increase in breadth of the DOS reflects the increased degree of incommensurability of these pitch ratios where the configurational energy landscape becomes much flatter. As the pitch ratio approaches the commensurate condition of $\sqrt{3}$, the distribution narrows and there is a high-angle preferential twist angle, which is close to $30^{\circ}$.

Shown in Figure 3d,e,f are the simulated minimum energy configurations for pitch ratios of $0.87,1.13$ and 1.60 where the local relative rotations are mapped to each dot (the lattice strains are mapped in Figure 3g,h,i). These simulated lowest-energy configurations are strikingly similar to the corresponding experimentally observed configurations (Figure 2), where the patterns are composed of arrays of pseudo-commensurate triangular domains connected by nodes where the lattice is both locally rotated and strained. To quantitatively predict whether SDWs occur at all pitch ratios, which can be either transverse (local lattice rotation) or longitudinal (local lattice strain), the standard deviations of the distributions local lattice rotation and local lattice strain were determined for each relaxed configuration and mapped in Figure 3b,c. As seen from these maps, there is clearly strong SDW formation at pitch ratios within the vicinity of 
commensurate conditions, but near the maximally incommensurate pitch ratios $(\sim 1.3-$ 1.5) there does not appear to be any significant SDW formation, as indicated by the low variance of local relative rotation and strain for these pitch ratios. It is also noted that the maps of angle change and strain are not identical, as the system tends to preferentially form transverse SDWs at twist angles farther away from a commensurate condition. Nonetheless, the final configuration is generally composed of SDWs that are a mixture of both transverse and longitudinal modes.
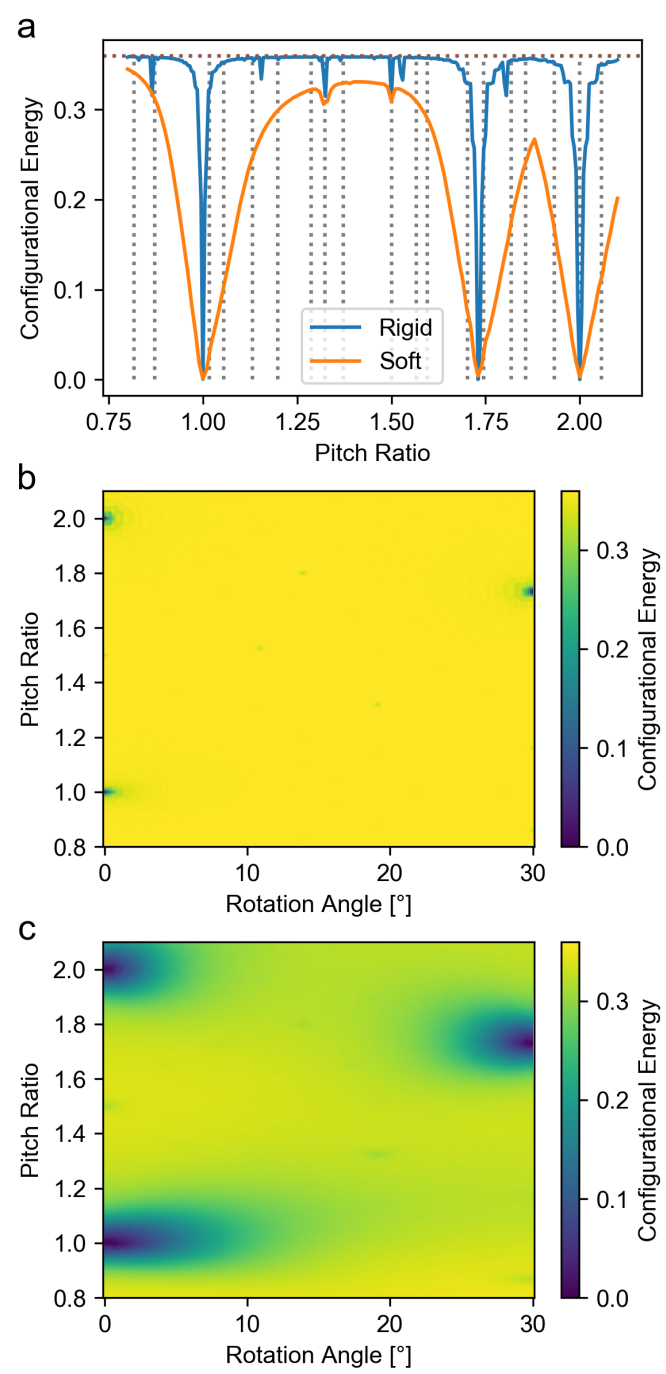

Figure 4. Minimum configurational energy vs pitch ratio from simulation. a, Minimum configurational energies available to a rigid epilayer (blue) allowing only global rotations and shifts and a soft epilayer lattice (orange) allowing global rotations, shifts and strain as well as local rotation and strain. The dotted horizontal line at $\sim 0.36$ represents the energy of a random dot configuration and the vertical dotted lines are the pitch ratios of samples produced in the study to compare with experimentally determined 
values. b,c, Value maps of the configurational energy of dot pattern formation versus the pitch ratio and rotation angle of the starting state for $\mathbf{b}$ a rigid $\mathbf{c}$ soft lattice.

From the above experimental and simulation data, it is clear that for most incommensurate pitch ratios there is a preferential twist angle and local lattice relaxations in the form of SDWs. However, the causal relationship between SDWs and preferential angle is unclear: do SDWs form as a result of a second-order energy minimization mechanism from an already low energy configuration of a pair of twisted lattices or do preferential angles occur in order to allow for the formation of SDWs? This question is answered by simulations in which the same energy minimization process is performed as above, but instead, the pair-potential of the epilayer is adjusted such that it is perfectly rigid, only allowing for global twisting and translation. Shown in Figure 4a is a plot of the minimum configurational energy for such a rigid epilayer, as a function of pitch ratio.

For commensurate conditions of (pitch ratios)@(twist angle) of $1 @ 0^{\circ}, \sqrt{3} @ 30^{\circ}$ and $2 @ 0^{\circ}$, it can be seen that the rigid epilayers achieve the lowest possible configurational energy of zero, which corresponds to all epi-dots being located in the interstitial positions of the bottom lattice. There are also shallow, but sharp dips in the configurational energy

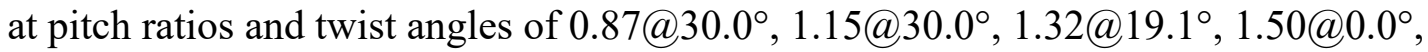
1.53@10.9, $1.80 @ 13.9^{\circ}\left(\sqrt{3} / 2 @ \tan ^{-1}(\sqrt{3} / 3), \sqrt{12} / 3 @ \tan ^{-1}(\sqrt{3} / 3), \sqrt{7} /\right.$ $\left.2 @ \tan ^{-1}(\sqrt{3} / 5), \sqrt{9} / 2 @ \tan ^{-1}(0), \sqrt{21} / 3 @ \tan ^{-1}(\sqrt{3} / 9), \sqrt{13} / 2 @ \tan ^{-1}(\sqrt{3} / 7)\right)$. These dips correspond to the so-called higher-order commensurate configurations, where a non-zero fraction of dots occupy perfect interstitial positions. Select higher-order commensurate configurations are shown in Figure S8, and a complete mapping of all possible higher-order commensurate structures is shown in Figure S9. Outside of the commensurate and a small number of higher-order commensurate pitch ratios, all other pitch ratios of the rigid epilayer possess a minimum configurational energy approaching $(\pi-4 / \pi) / \sqrt{27} \sim 0.36$, which corresponds to the configurational energy of a maximally incommensurate epilayer (see derivation in the Supplementary Information). These simulations imply that for all pitch ratios, except a small finite set of commensurate and higher-order commensurate pitch ratios, the configurational energy is essentially invariant to global twisting of the epilayer (Figure 4b). Conversely, for a soft epilayer, there are significant reductions of the configurational energy at all incommensurate pitch 
ratios, relative to the rigid epilayer (Figure 4a,c). Therefore, given that soft epilayers generally adopt a twist angle, while rigid epilayers are invariant to global twisting, it is concluded that experimentally observed twist angles in soft epilayers occur as a consequence of SDW formation; structural relaxation is dominated by SDW formation (opposed to higher-order commensuration) whose configurational energy is strongly modulated by a global twisting of the epilayer. Put another way, SDWs are not a secondorder energy minimization process on top of higher-order commensuration; formation of SDWs is a primary mechanism of energy minimization for soft-hard epitaxial systems.

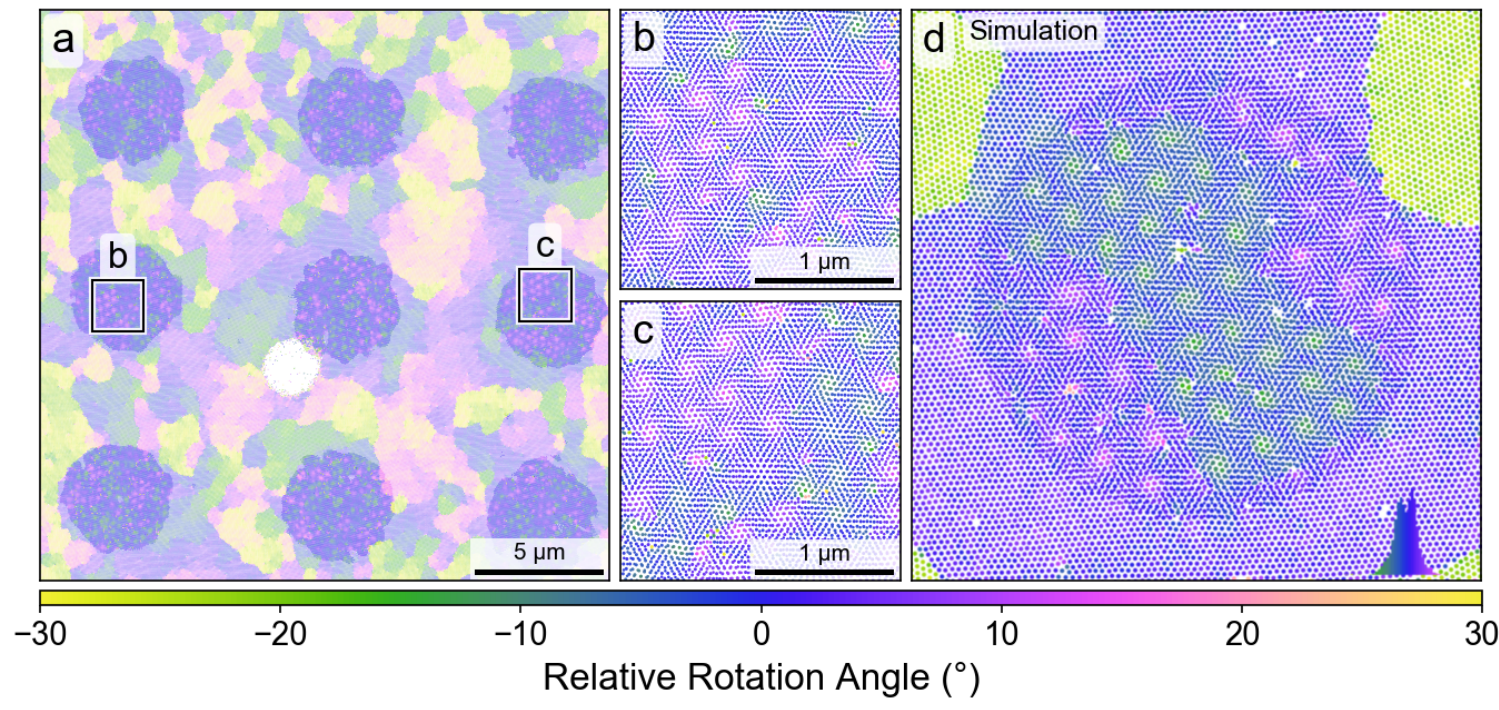

Figure 5. Comparison between experiment and MD simulation of epitaxial BCP dot patterns. a, Large-area relative rotation angle map for a pitch ratio of 1.13. b,c, Selected areas as indicated in $a$ showing of EBL patterned arrays showing both the EBL posts and BCP dots. $d$, Simulated dot positions for pitch ratio of 1.13 mapped with their local relative rotation angle as indicated in the histogram. See the Supplementary Information for an animation of the simulated annealing.

To better understand the kinetics of SDW pattern formation, molecular dynamics simulations at a pitch ratio of 1.13 for a soft-hard epitaxial system were performed. Briefly, all potentials used are similar as previously described for the energy minimization study, a canonical ensemble (NVT) is used, where the system temperature is controlled using a Nose-Hoover thermostat, and periodic boundary conditions are applied (see Supplementary Information for more details). As shown in Supplementary Video 1, the soft epilayer is initially brought to a sufficiently high temperature to create a homogenous melt. As the system temperature is slowly decreased, crystallization occurs 
and hexagonally packed grains with random orientations become visible outside of the patterned registration layer. Within the patterned registration region, crystallization also occurs, but this process is frustrated by the mismatch in lattice parameters. In order to accommodate the competition between interlayer registration and intralayer closepacking, nodes of local structural relaxation occur in the form of SDWs. As the microstructure evolves over time, two low energy SDW configurations can be seen, which correspond to local relative rotations of ca. $\pm 10^{\circ}$. Moreover, the SDW nodes themselves tend to aggregate in pseudo-hexagonally packed regions of either ca. $+10^{\circ}$ or $-10^{\circ}$ (Figure 5d). Shown in Figure 5a is a large-area mapping of local relative rotation of a BCP film that has been annealed on an EBL patterned substrate, where the patterned post arrays can be clearly identified by their preferential angle relative to the uniform background of random grain orientations. Closer inspection of these post arrays (Figure 5b,c) reveals a morphology strikingly similar to that found in the simulations, where the BCP has undergone significant local relaxations, forming SDWs nodes at relative rotations of ca. $\pm 10^{\circ}$, which are also aggregated in pseudo-close-packed regions of SDWs with the same orientations. The excellent agreement of these simulations with experimental data suggests that the very simple physics of interlayer registration competing with intralayer close-packing explains the observed structural relaxations and SDW formation observed in dot forming BCPs on an incommensurate template.

The recent discovery of superconductivity and other remarkable properties arising from magic-angle twisted bilayer graphene has resulted in an explosion of interest in the properties of topological twisted van der Waals bilayer materials. A fundamentally important phenomenon to understanding the properties of these topological materials is local structural relaxation that can occur as a result of epitaxial lattice mismatch. However, structural relaxation of epitaxial 2-dimensional atomic materials can be notoriously difficult to study using direct methods for displacements measured in fractions of Angstroms. The self-assembly of dot forming block copolymers on a prepatterned hexagonal array of shallow posts can be used as a model system to systematically explore the mechanisms and structural variety of relaxation modes in 2dimensional epitaxial systems. With incommensurate pitch ratios, it is found that the resulting epitaxial patterns are ubiquitously dominated by the formation of SDWs. 
Molecular dynamics simulations demonstrate that preferential twist angles of these systems result from specific SDW configurations that minimize the energy of the system. Given the fundamentally important nature of both the twist angle and the local relaxation of the epilayer for the electronic properties of van der Waals bilayer materials, these insights may aid in the rational engineering for the self-assembly of bilayer materials with specific structure-property relationships.

\section{Methods}

\section{Materials}

PS- $b$-PDMS (31K-14.5K, PDI 1.15) and polystyrene (10K, PDI 1.09) were obtained from Polymer Source Incorporated. Silicon wafers $(<100>$, p-type, resistivity $<0.005 \Omega \cdot \mathrm{cm}, 4$ inches in diameter, $525 \pm 25 \mu \mathrm{m}$ in thickness) were purchased from WRS materials. Sulfuric acid (96\%), hydrogen peroxide (30\%), and tetramethylammonium hydroxide (TMAH, 25\% in water) were obtained from Avantor Performance Materials. Toluene (99.9\%) was purchased from Fisher Scientific. Methyl isobutyl ketone (MIBK, >99.5\%), tetrahydrofuran (THF, 99.9\%), and syringe filters (Whatman Puradisc, PTFE membrane with $0.1 \mu \mathrm{m}$ pore size) were purchased from Sigma-Aldrich. Glass syringes were obtained from Cadence Science Incorporated. Negative tone electron beam resist hydrogen silsesquioxane (HSQ) was donated by the group of Prof. Jonathan G. C. Veinot (Department of Chemistry, University of Alberta). All chemicals and materials were used as received.

\section{EBL post array preparation}

4-inch silicon wafers were diced into $1 \times 1 \mathrm{~cm}$ chips with a DSA 321 dicing saw. The diced silicon chips were cleaned in piranha solution (3:1 volume ratio of $96 \%$ sulfuric acid and 30\% hydrogen peroxide) for 15 min before washing in deionized water (Millipore) and dried in a nitrogen stream. Silicon chips were then baked at $180 \mathrm{oC}$ on a hot plate for 10 min to ensure they were completely dried. A MIBK solution of HSQ with a concentration of $0.65 \mathrm{wt} \%$ was prepared and filtered right before use. HSQ films were spin-cast for 60 seconds at $8000 \mathrm{rpm}$ with a $1.5 \mathrm{sec}$ ramp. All HSQ films were transferred 
into the EBL vacuum system right after spin-casting without baking. The EBL parameters were: $30 \mathrm{kV}, 9 \mathrm{~cm}$ working distance, $10 \mu \mathrm{m}$ aperture. Circular arrays of posts were patterned with varying of circles for each pitch: the $30 \mathrm{~nm}$ pitch had 25 arrays, the 36 and $38 \mathrm{~nm}$ pitches had 20 arrays; the 44 and $46 \mathrm{~nm}$ pitches had 16 arrays; the $50 \mathrm{~nm}$ pitch had 20 arrays; the $52-90 \mathrm{~nm}$ pitch had 15 arrays (these correspond to pitch ratios of $0.68,0.82 \& 0.87,1.02 \& 1.05,1.13,1.20-2.06$, respectively). The number of posts also varied, with the 30 to $38 \mathrm{~nm}$ pitches having about $3000 \mathrm{HSQ}$ posts per circle, the remaining post arrays contained about $6500 \mathrm{HSQ}$ posts per circle. The height of the posts was then measured using a Veeco Multimode AFM (see Figure S1).

\section{Block copolymer thin film self-assembly}

PS- $b$-PDMS (31k-14.5k)/30\% PS (10K) toluene solution was prepared by mixing 1 PS- $b$ PDMS (31k-14.5k) toluene solution and $1 \mathrm{wt} . \%$ PS (10k) toluene solution in a 7:3 ratio. This BCP solution was then spin-cast on EBL-patterned silicon chips at $5000 \mathrm{rpm}$ for 40 s. BCP thin film thicknesses were about $30 \mathrm{~nm}$, as measured by AFM. BCP thin films were annealed in our static solvent vapor annealing system to reach a swelling degree of 2.2 (see reference ${ }^{34}$ for a more detailed description). Annealed BCP thin films were converted into silica dots arrays with a two-step RIE etching procedure: $1 . \mathrm{CF}_{4}, 100$ mTorr, $50 \mathrm{~W}, 10 \mathrm{~s} ; 2.80 \% / 20 \% \mathrm{O}_{2} / \mathrm{CF}_{4}, 145 \mathrm{mTorr}, 30 \mathrm{w}, 40 \mathrm{~s}$.

\section{Imaging}

All images were taken with a Hitachi S4800 scanning electron microscope (15 kV, 20 $\mu \mathrm{A})$ and a Zeiss Helium Ion Microscope (30.8 kV, $10 \mu \mathrm{m}$ aperture, spot size 4).

\section{Data processing}

BCP dot and EBL post positions were measured separately, from SEM micrographs. The dots and posts were separated and measured using FFT filtering on the SEM micrographs using Gwyddion. ${ }^{48}$ From the BCP dot positions the rotation angle and lattice strain were measured locally at each dot using its six nearest neighbors as reference. The local rotation angle was subtracted by the mean angle of the EBL posts to account for micrograph misalignment and the strain was measured with reference to the mean pitch 
of the unstrained dots directly outside the EBL post patterned regions. In the dot map visualizations, angles and strains are mapped onto the EBL posts using values from the nearest neighbor BCP dot to increase the color contrast in the maps. See Supplementary Information for more details on how these metrics were calculated.

\section{Simulation details}

Molecular dynamics simulations were performed using LAMMPS versions 12 Dec 2018 for the minimization and 20 Nov 2019 for the annealing from a melt. ${ }^{49}$ The simulations were performed in 2-dimensions with 2 sets of particles both forming hexagonal lattices, with periodic boundary conditions. One set of particles, acting as the EBL posts, was fixed in position and was not allowed to deform or move during the simulation. This fixed layer was cropped to a circle in the center of the simulation area with padding on the side to allow the other particle group to act as the $\mathrm{BCP}$ dots to relax to a regular hexagonal structure before the boundary. There were two potentials acting on the BCP particles: a cosine interlayer potential that allowed for overlap with the fixed ebeam layer and a soft morse potential added to a strong Lennard-Jones potential to represent the soft $\mathrm{BCP}$ intralayer potential with a harder BCP center. The hard repulsive portion of the Lennard-jones potential only affected the annealing results by preventing particle overlap during high-temperature melting. The simulations to make the maps of different pitch ratios and angles as seen in Figure 3, were performed by rotating and scaling the bottom lattice representing the ebeam posts and performing a minimization on the system energy by allowing the top BCP lattice to relax. The energy minimization was implemented using the Polak-Ribiere version of the conjugate gradient algorithm included in the LAMMPS package. The final minimized energy is reported as a function of the initial starting configuration. Since the top layer is allowed to deform, this can affect the global rotation and scale of the final configuration, however, shown in Figure S7c and $d$ the change of the global scale and rotation was found to be less than $\pm 1 \%$ and $\pm 1^{\circ}$ respectively. The molecular dynamics simulations use similar potentials, where the initial temperature is sufficiently high to induce melting, which randomizes the particle starting positions. Following this, the system is annealed at a moderate temperature for some time and then allowed to cool to a lower temperature. See the Supplementary material for 
more details on the statistical calculations, example LAMMPS files and the particle pair potentials.

\section{Data availability}

The data that support the findings of this study are available from the corresponding author on reasonable request.

\section{Acknowledgments}

This work was supported by Alberta Innovates Technology Futures (graduate fellowship to CJ, and grant numbers CTDP-G2018000919 and AITF iCORE IC50-T1 G2013000198), the Natural Sciences and Engineering Research Council (NSERC, grant numbers RGPIN-2014-05195 and RGPIN 2019-04346), and the Canada Research Chairs Program (CRC 207142). Dr. Jon Veinot is thanked for the donation of HSQ E-beam resist. Dr. Aaron Hryciw and Peng Li are thanked for valuable assistance with the EBL fabrication and HiM imaging at the University of Alberta Centre for Nanofabrication.

\section{Author Information}

\section{Affiliations}

Department of Chemistry, University of Alberta, Edmonton, AB T6G 2G2, Canada

Cong Jin, Brian C. Olsen, Erik J. Luber \& Jillian M. Buriak

National Research Council Canada, Nanotechnology Research Centre, Edmonton, AB T6G 2M9, Canada

Cong Jin, Brian C. Olsen, Erik J. Luber \& Jillian M. Buriak

\section{ORCIDs}

Cong Jin: 0000-0002-6996-054X

Brian C. Olsen: 0000-0001-9758-3641

Erik J. Luber: 0000-0003-1623-0102

Jillian M. Buriak: 0000-0002-9567-4328 


\section{Contributions}

C.J. performed all experimental work: e-beam lithography, $\mathrm{BCP}$ deposition, annealing and etching, HiM imaging. B.C.O. and E.J.L. wrote python code for data analysis, performed image analysis, LAMMPS simulations, and analytical derivations. C.J., B.C.O., E.J.L., and J.M.B. conceived of the experiments and wrote the paper.

\section{Corresponding Authors}

Correspondence to Jillian Buriak (E-mail: jburiak@ualberta.ca) or Erik Luber (E-mail: eluber@ualberta.ca)

\section{Ethics declarations}

\section{Competing interests}

The authors declare no competing interests.

\section{Supplementary information}

Supplementary Figs. 1-15 and discussion.

\section{References}

1. Bedoya-Pinto, A. et al. Realization of Epitaxial NbP and TaP Weyl Semimetal Thin Films. ACS Nano 14, 4405-4413 (2020).

2. Fu, W. et al. Room Temperature Commensurate Charge Density Wave on Epitaxially Grown Bilayer 2H-Tantalum Sulfide on Hexagonal Boron Nitride. ACS Nano 14, $3917-$ $3926(2020)$.

3. Bae, S.-H. et al. Integration of bulk materials with two-dimensional materials for physical coupling and applications. Nat. Mater. 18, 550-560 (2019).

4. Nakano, M. et al. Intrinsic 2D Ferromagnetism in $\mathrm{V}_{5} \mathrm{Se}_{8}$ Epitaxial Thin Films. Nano Lett. 19, 8806-8810 (2019). 
5. Zhao, W. et al. Magnetic proximity and nonreciprocal current switching in a monolayer $\mathrm{WTe}_{2}$ helical edge. Nat. Mater. 19, 503-507 (2020).

6. Wei, P. et al. Strong interfacial exchange field in the graphene/EuS heterostructure. Nat. Mater. 15, 711-716 (2016).

7. Zhong, D. et al. Van der Waals engineering of ferromagnetic semiconductor heterostructures for spin and valleytronics. Sci. Adv. 3, e1603113 (2017).

8. Miao, J. et al. Gate-Tunable Semiconductor Heterojunctions from 2D/3D van der Waals Interfaces. Nano Lett. 20, 2907-2915 (2020).

9. Jariwala, D., Marks, T. J. \& Hersam, M. C. Mixed-dimensional van der Waals heterostructures. Nat. Mater. 16, 170-181 (2017).

10. Gu, Z.-G., Chen, S.-C., Fu, W.-Q., Zheng, Q. \& Zhang, J. Epitaxial Growth of MOF Thin Film for Modifying the Dielectric Layer in Organic Field-Effect Transistors. ACS Appl. Mater. Interfaces 9, 7259-7264 (2017).

11. Janneck, R. et al. Highly Crystalline C8-BTBT Thin-Film Transistors by Lateral Homo-Epitaxial Growth on Printed Templates. Adv. Mater. 29, 1703864 (2017).

12. Zwick, C., Meissner, M., Sojka, F., Forker, R. \& Fritz, T. In-depth characterization of annealing-induced restructuring processes of doped organic adlayers. Phys. Rev. Mater. 3, 085604 (2019).

13. Nakayama, Y. et al. Epitaxial Growth of an Organic p-n Heterojunction: C60 on Single-Crystal Pentacene. ACS Appl. Mater. Interfaces 8, 13499-13505 (2016).

14. Li, D.-J. et al. Epitaxial Growth of Oriented Metalloporphyrin Network Thin Film for Improved Selectivity of Volatile Organic Compounds. Small 13, 1604035 (2017).

15. Liu, X. et al. Epitaxial Ultrathin Organic Crystals on Graphene for High-Efficiency Phototransistors. Adv. Mater. 28, 5200-5205 (2016).

16. Cao, M. et al. Enhanced photoelectrical response of thermodynamically epitaxial organic crystals at the two-dimensional limit. Nat. Commun. 10, 1-11 (2019). 
17. Yuan, L. et al. Twist-angle-dependent interlayer exciton diffusion in $\mathrm{WS}_{2}-\mathrm{WSe}_{2}$ heterobilayers. Nat. Mater. 1-7 (2020).

18. Hod, O., Meyer, E., Zheng, Q. \& Urbakh, M. Structural superlubricity and ultralow friction across the length scales. Nature 563, 485-492 (2018).

19. Cao, Y. et al. Unconventional superconductivity in magic-angle graphene superlattices. Nature 556, 43-50 (2018).

20. Yoo, H. et al. Atomic and electronic reconstruction at the van der Waals interface in twisted bilayer graphene. Nat. Mater. 18, 448-453 (2019).

21. Lian, B., Wang, Z. \& Bernevig, B. A. Twisted Bilayer Graphene: A Phonon-Driven Superconductor. Phys. Rev. Lett. 122, 257002 (2019).

22. Yankowitz, M. et al. Tuning superconductivity in twisted bilayer graphene. Science 363, 1059-1064 (2019).

23. Rosenberger, M. R. et al. Twist Angle-Dependent Atomic Reconstruction and Moiré Patterns in Transition Metal Dichalcogenide Heterostructures. ACS Nano 14, 4550-4558 (2020).

24. Song, T. et al. Switching 2D magnetic states via pressure tuning of layer stacking. Nat. Mater. 18, 1298-1302 (2019).

25. Lin, Y.-P. \& Nandkishore, R. M. Chiral twist on the high- $T_{\mathrm{c}}$ phase diagram in moiré heterostructures. Phys. Rev. B 100, 085136 (2019).

26. Merkl, P. et al. Ultrafast transition between exciton phases in van der Waals heterostructures. Nat. Mater. 18, 691-696 (2019).

27. Lee, J. Y. et al. Theory of correlated insulating behaviour and spin-triplet superconductivity in twisted double bilayer graphene. Nat. Commun. 10, 1-10 (2019).

28. Koshino, M. et al. Maximally Localized Wannier Orbitals and the Extended Hubbard Model for Twisted Bilayer Graphene. Phys. Rev. X 8, 031087 (2018). 
29. Nam, N. N. T. \& Koshino, M. Lattice relaxation and energy band modulation in twisted bilayer graphene. Phys. Rev. B 96, 075311 (2017).

30. Last, J. A., Hooks, D. E., Hillier, A. C. \& Ward, M. D. The Physicochemical Origins of Coincident Epitaxy in Molecular Overlayers: Lattice Modeling vs Potential Energy Calculations. J. Phys. Chem. B 103, 6723-6733 (1999).

31. Meissner, M. et al. Flexible 2D Crystals of Polycyclic Aromatics Stabilized by Static Distortion Waves. ACS Nano 10, 6474-6483 (2016).

32. Novaco, A. D. \& McTague, J. P. Orientational Epitaxy-the Orientational Ordering of Incommensurate Structures. Phys. Rev. Lett. 38, 1286-1289 (1977).

33. Sushko, A. et al. High resolution imaging of reconstructed domains and moire patterns in functional van der Waals heterostructure devices. (2019).

34. Jin, C., Olsen, B. C., Wu, N. L. Y., Luber, E. J. \& Buriak, J. M. Sequential Nanopatterned Block Copolymer Self-Assembly on Surfaces. Langmuir 32, 5890-5898 (2016).

35. Bita, I. et al. Graphoepitaxy of Self-Assembled Block Copolymers on TwoDimensional Periodic Patterned Templates. Science 321, 939-943 (2008).

36. Jin, C., Olsen, B. C., Luber, E. J. \& Buriak, J. M. Preferential Alignment of Incommensurate Block Copolymer Dot Arrays Forming Moiré Superstructures. ACS Nano 11, 3237-3246 (2017).

37. Shaw, C. G., Fain, S. C. \& Chinn, M. D. Observation of Orientational Ordering of Incommensurate Argon Monolayers on Graphite. Phys. Rev. Lett. 41, 955-957 (1978).

38. Gärtner, S., Fiedler, B., Bauer, O., Marele, A. \& Sokolowski, M. M. Lateral ordering of PTCDA on the clean and the oxygen pre-covered $\mathrm{Cu}(100)$ surface investigated by scanning tunneling microscopy and low energy electron diffraction. Beilstein J. Org. Chem. 10, 2055-2064 (2014). 
39. Kilian, L. et al. The commensurate-to-incommensurate phase transition of an organic monolayer: A high resolution LEED analysis of the superstructures of NTCDA on $\operatorname{Ag}(111)$. Surf. Sci. 602, 2427-2434 (2008).

40. Huempfner, T., Sojka, F., Forker, R. \& Fritz, T. Growth of coronene on (100)- and (111)-surfaces of fcc-crystals. Surf. Sci. C, 80-88 (2015).

41. Plimpton, S. Fast Parallel Algorithms for Short-Range Molecular Dynamics. $J$. Comput. Phys. 117, 1-19 (1995).

42. Rahman, A., Majewski, P. W., Doerk, G., Black, C. T. \& Yager, K. G. Non-native three-dimensional block copolymer morphologies. Nat. Commun. 7, 13988 (2016).

43. Woods, C. R. et al. Commensurate-incommensurate transition in graphene on hexagonal boron nitride. Nat. Phys. 10, 451-456 (2014).

44. Kim, N. Y. et al. Evidence of Local Commensurate State with Lattice Match of Graphene on Hexagonal Boron Nitride. ACS Nano 11, 7084-7090 (2017).

45. Wijk, M. M. van, Schuring, A., Katsnelson, M. I. \& Fasolino, A. Relaxation of moiré patterns for slightly misaligned identical lattices: Graphene on graphite. 2D Mater. 2 , 034010 (2015).

46. Gargiulo, F. \& Yazyev, O. V. Structural and electronic transformation in low-angle twisted bilayer graphene. 2D Mater. 5, 015019 (2017).

47. Dai, S., Xiang, Y. \& Srolovitz, D. J. Twisted Bilayer Graphene: Moiré with a Twist. Nano Lett. 16, 5923-5927 (2016).

48. Něcas, D. \& Klapetek, P. Gwyddion: An open-source software for SPM data analysis. Cent. Eur. J. Phys. 10, 181-188 (2012).

49. Plimpton, S. Fast Parallel Algorithms for Short-Range Molecular Dynamics. $J$. Comput. Phys. 117, 1-19 (1995). 